\title{
Biologia reprodutiva de Hexanematichthys proops (Siluriformes, Ariidae) no litoral ocidental maranhense
}

\author{
Geuza Cantanhêde ${ }^{1}$, Antonio C. L. de Castro ${ }^{2} \&$ Éder A. Gubiani ${ }^{1}$
}

1. Curso de Pós-Graduação em Ecologia de Ambientes Aquáticos Continentais, Departamento de Biologia, Universidade Estadual de Maringá, Avenida Colombo, 5970 - Campus Universitário, 87020-900 Maringá, Paraná, Brasil. (geuzac@yahoo.com.br) (eagubiani@nupelia.uem.br)

2. Departamento de Oceanografia e Limnologia, Universidade Federal do Maranhão, Avenida dos Portugueses, S/N - Campus do Bacanga, 65085-580 São Luís, Maranhão, Brasil. (alec@ufma.br)

\begin{abstract}
Reproductive biology of Hexanematichthys proops (Siluriforme, Ariidae) in the western coast of Maranhão. The aim of this study was to investigate the influence of gonadal and nutritional indexes on the reproductive biology of Hexanematichthys proops (Valenciennes, 1840) (Siluriformes, Ariidae) in the western coast of Maranhão, between July 2001 and July 2002 . A significant female dominance was observed during the study period. The weight/length relationship showed a significant difference between sexes in relation to the alometric coefficient, although both sexes showed positive alometric growth. Analysis of the maturation curve based on the monthly variation of the gonadosomatic and hepatosomatic index suggests that the reproductive period occurs between October and May. The month variation of the condition factor reveals that females presented general conditions poorer than males during all the study period. It was observed that the loss of liver weight occurs during gonadal maturation and reproductive processes. Analysis of the mean repletion index showed that the fishes exhibited better feeding conditions before and after the reproductive season.
\end{abstract}

KEYWORDS. Ratio sex, weight/length relationship, reproduction, Hexanematichthys proops.

RESUMO. Objetivou-se investigar a influência dos índices gonadais e nutricionais sobre a biologia reprodutiva de Hexanematichthys proops (Valenciennes, 1840) (Siluriformes, Ariidae) na costa ocidental do Maranhão entre julho de 2001 e julho de 2002. Houve uma significante dominância de fêmeas quando considerado o período total amostrado. A relação peso/comprimento apresentou uma significante diferença entre os sexos em relação ao coeficiente de alometria, embora ambos tenham apresentado crescimento alométrico positivo. Análises da curva de maturação baseadas na variação mensal dos índices gonadossomático e hepatossomático sugerem que o período reprodutivo ocorre entre outubro e maio. A variação mensal do fator de condição revelou que as fêmeas apresentaram condições gerais inferiores aos machos durante todo o período amostral. Foi observado que a diminuição do peso do fígado ocorre durante a maturação gonadal e processos reprodutivos. Análises do índice de repleção médio mostraram que os peixes exibem melhores condições alimentares antes e depois do período reprodutivo.

PALAVRAS-CHAVE. Proporção sexual, relação peso/comprimento, reprodução, Hexanematichthys proops.

Hexanematichthys proops (Valenciennes, 1840), comumente conhecido por uritinga, é um bagre marinho de grande importância nas atividades pesqueiras, em especial na pesca artesanal. Diversos trabalhos relatam a importância de bagres como recurso pesqueiro em todo o litoral brasileiro, seja pelo seu valor econômico ou como recurso de subsistência para a população humana (MishIMA \& TANII, 1982; ARAúJO, 1984; REIS, 1986; BARBIERI et al., 1992).

Os peixes denominados bagres marinhos pertencem à família Ariidae e ocorrem nas zonas litorâneas tropicais e subtropicais em ambientes marinhos, estuarinos e de água doce, sendo geralmente abundantes em águas costeiras com fundo lodoso e pouco profundas (ARAÚJo, 1988; ANDREATA et al., 1989). Esses peixes geralmente procuram a embocadura dos rios e regiões lagunares na época da desova (Figueiredo \& Menezes, 1978; Moyle \& CECH-JR., 1988).

A espécie $H$. proops apresenta ampla distribuição, ocorrendo em toda a América do Sul e Ilhas do Caribe em rios que drenam para o Atlântico e em estuários da Colômbia ao Brasil (ReIs et al., 2003). Na Guiana Francesa é uma espécie comum em estuários, apresentando um rápido crescimento e curta longevidade, sendo uma importante fonte econômica e alimentar para a população humana local (Planquette \& Rojas-Beltran, 1984).
Os bagres Ariidae têm sido amplamente estudados no Brasil em trabalhos que abordam aspectos como alimentação (Araúso, 1984; Reis, 1986; Melo \& TeIXeIra, 1992; Pedra et al., 2006), distribuição (CRAIG, 1980; Mishima \& TANJi, 1981; Araújo 1988; AzEVEdo et al., 1999), idade e crescimento (Oliveira \& Novelli, 2005) e reprodução (BARBIERI et al., 1992; CHAVES, 1994; GOMES \& Araúso, 2004; FÁvaro et al., 2005). Hexanematichthys proops, no entanto, foi muito pouco estudado, a despeito de sua reconhecida importância econômica. Os poucos trabalhos publicados sobre a espécie foram realizados na Guiana Francesa (LeCOMTE et al., 1986; 1989; RoJAsBeltran, 1989; Meunier et al., 1994). No Brasil há apenas um estudo relacionado à alimentação da espécie na Ilha de Itamaracá, PE (Guedes \& VAsconcelos Filho, 1980).

O desenvolvimento de programas de preservação de estoques naturais de peixes, em especial daqueles explorados comercialmente, depende de estudos da biologia das espécies, os quais são feitos através de investigação dos parâmetros biológicos inerentes à dinâmica populacional (Goulart \& Verani, 1992). Por exemplo, a interpretação das variações do fator de condição, o qual é um indicador quantitativo do grau de higidez ou de bem estar do peixe pode refletir condições alimentares recentes (LE-CREN, 1951; V AZZOLER, 1996). O 
índice gonadossomático (IGS), o qual reflete o desenvolvimento dos ovários como porcentagem do peso total de maneira crítica, pode indicar quantitativamente o grau de desenvolvimento gonadal e a época de desova (VAZZOLER et al., 1989).

Com o objetivo de investigar a biologia reprodutiva de $H$. proops, o presente trabalho aborda as relações entre algumas variáveis biológicas dessa espécie associadas ao estado nutricional e ao seu ciclo reprodutivo.

\section{MATERIAL E MÉTODOS}

A costa do Maranhão possui uma extensão de 640 $\mathrm{km}$, sendo caracterizada por uma série de baías e estuários ligados por canais naturais através dos mangues. A plataforma continental é larga e apenas ligeiramente inclinada e as águas costeiras são rasas e sob influência da descarga de um grande número de rios. A área de estudo compreende as zonas costeiras e estuarina de parte do litoral ocidental maranhense (latitudes de $1^{\circ} 20^{\prime} \mathrm{S}$ e $2^{\circ} 32^{\prime}$ S ; longitudes de $44^{\circ} 18^{\prime} \mathrm{W}$ e $45^{\circ} 20^{\prime} \mathrm{W}$ ), cobrindo a região que vai da baía de São Marcos até a baía de Turiaçu (Fig. 1).

Os peixes foram coletados em nove amostragens no período de julho de 2001 a julho de 2002 em seis áreas de pesca na costa ocidental maranhense: Apeuzinho, Atim, Urumaru, Muricitil, Pericaua e Porto Rico (Fig. 1). Foram utilizados dois tipos de redes de emalhar, uma delas popularmente conhecida como malhadeira ou pescadeira (com malhas entre 175 e 200mm). O tamanho dessas redes variou de 90 a $2.500 \mathrm{~m}$ de comprimento e de 2,8 a $3,5 \mathrm{~m}$ de altura. As redes foram lançadas em canais e igarapés mais profundos nos quais também se captura a pescada amarela (Cynoscion acoupa (Lacepède, 1801)), camurins (Centropomus paralellus Poey, 1860 e C. undecimalis (Bloch, 1792)) e diversas espécies de cação, entre outros. A outra rede, conhecida como serreira, é uma rede de emalhar de deriva flutuante que tem como espécie alvo o peixe serra (Scomberomurus brasiliensis Collette, Russo \& Zavala-Camin, 1978). Entretanto, captura outros peixes, inclusive uritinga. Essa rede apresenta tamanho de malha entre 95 e $100 \mathrm{~mm}$, com comprimento variando de 1.100 a $1.650 \mathrm{~m}$ (dependendo do tamanho da embarcação e com aproximadamente $4 \mathrm{~m}$ de altura).

Em laboratório registrou-se o peso total (Wt), comprimento total (Lt), peso do estômago (We), sexo, peso das gônadas (Wg) e do fígado (Wf) de cada exemplar de $H$. proops. Os dados de comprimento tiveram acuracidade de milímetros e os de peso, de gramas.

A proporção sexual foi determinada pelas frequiências mensais e total de machos e fêmeas coletados. Aplicou-se o teste do qui-quadrado $\left(\chi^{2}\right)$ em nível de $5 \%$ de significância para detectar possíveis diferenças significativas nessas proporções.

A relação entre o peso e o comprimento total para cada sexo foi estabelecida pela equação: $\mathrm{W}_{t}=\mathrm{a} \cdot \mathrm{L}_{\mathrm{t}}{ }^{\mathrm{b}}$ (DOS SAntos, 1978), onde $\mathrm{W}_{\mathrm{t}}$ é o peso total, $\mathrm{L}_{\mathrm{t}}$ é o comprimento total, $a$ é fator de condição relacionado com o grau de engorda e $b$ é a constante relacionada com o tipo de crescimento dos indivíduos.

Para estimar os parâmetros da relação peso/ comprimento foi utilizado o programa Statistic ${ }^{\text {TM }}$ for Windows 7.1 (Statsoft Inc., 2005), através de procedimento não-linear com a utilização do algoritmo de Gauss-Newton (processo interativo que exige a

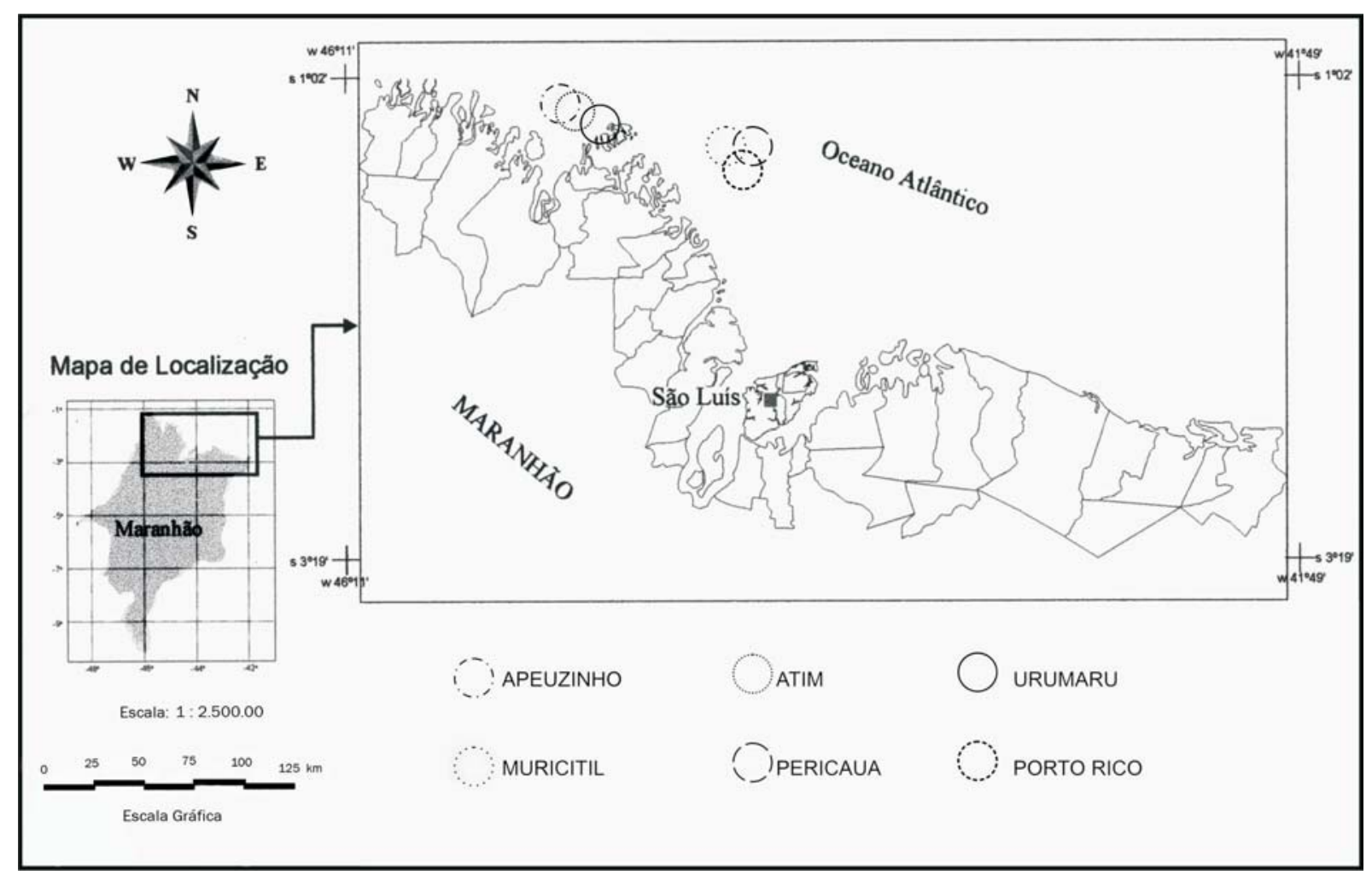

Fig. 1. Localização da área de amostragem no litoral maranhense. 
introdução de valores iniciais (sementes) para os parâmetros a serem estimados) (MYERs, 1990).

Para testar as diferenças entre os parâmetros das curvas ajustadas para machos e fêmeas, uma análise de ANCOVA (GolDBERG \& SCHEINER, 1993) foi aplicada aos parâmetros das regressões lineares do comprimento total e peso total transformados $(\log 10)$ em nível de significância de $5 \%$.

$\mathrm{O}$ fator de condição $(\mathrm{K})$ foi estimado para cada indivíduo através da expressão: $\mathrm{K}=\mathrm{W}_{\mathrm{t}} / \mathrm{L}_{\mathrm{t}}^{\mathrm{b}}$ (VAZZOLER, 1996), onde $b$ é o coeficiente angular da relação peso/ comprimento. Foram feitas análises gráficas da variação mensal dos valores médios do fator de condição.

O índice de repleção (IR) foi calculado para cada indivíduo através da seguinte relação: $\mathrm{IR}=\left(\mathrm{W}_{\mathrm{e}} / \mathrm{W}_{\mathrm{t}}\right) \times 100$ (ZaVAla-CAMin, 1996), onde $\mathrm{W}_{\mathrm{e}}$ é o peso do estômago.

O índice gonadossomático (IGS), utilizado para inferir acerca do ciclo reprodutivo e o hepatossomático (IHS) foram calculados como a razão entre o peso total e do órgão considerado, como segue: IGS $=\left(\mathrm{W}_{\mathrm{g}} / \mathrm{W}_{\mathrm{t}}\right)$ x 100 e IHS = $\left(\mathrm{W}_{\mathrm{f}} / \mathrm{W}_{\mathrm{t}}\right)$ x 100 (VAzzoler, 1996), onde $\mathrm{W}_{\mathrm{g}}$ é o peso das gônadas e $\mathrm{W}_{\mathrm{f}}$ é o peso do fígado.

\section{RESULTADOS E DISCUSSÃO}

Foram amostrados 150 indivíduos de $H$. proops (115 fêmeas e 35 machos). A proporção sexual apresentou diferenças significativas nos meses de julho, setembro e outubro de 2001 e fevereiro e junho de 2002 ( $\mathrm{p}<0,05)$ (quando as fêmeas predominaram numericamente). $\mathrm{O}$ número de machos não foi superior ao de fêmeas em nenhum mês (Tab. I). Para o número total de indivíduos capturados também as fêmeas foram significativamente predominantes (Tab. I), uma vez que a proporção sexual total foi de 3 fêmeas para cada macho.

NIKOLSKY (1963) afirma que a proporção sexual varia consideravelmente de espécie para espécie, mas que na maioria é de 1:1. Para as espécies pertencentes à família Ariidae, as fêmeas em geral predominam (ETCHEVERS, 1978; ReIs, 1986; FÁvaro et al., 2005). A estrutura em sexo de uma população pode representar uma adaptação ao suprimento alimentar, havendo um predomínio de fêmeas quando o alimento disponível é abundante (NIKOLSKY, 1969). BARBIERI et al. (1992) sugerem que, durante o

Tabela I. Proporção sexual mensal e total de fêmeas e machos de Hexanematichthys proops (Valenciennes, 1840) (com teste do quiquadrado) capturados no litoral ocidental maranhense de julho/ 2001 a julho/2002. ( $*^{*}=$ significante em nível de 0,$\left.05 ; \chi_{0,05}^{2}=3,841\right)$.

\begin{tabular}{|c|c|c|c|c|c|c|}
\hline \multirow[b]{2}{*}{ MÊS } & \multicolumn{2}{|c|}{ FÊMEAS } & \multicolumn{2}{|c|}{ MACHOS } & \multirow[b]{2}{*}{ TOTAL } & \multirow[b]{2}{*}{$\chi_{0,05}^{2}$} \\
\hline & $\mathrm{N}$ & $\%$ & $\mathrm{~N}$ & $\%$ & & \\
\hline julho/2001 & 25 & 89,29 & 3 & 10,71 & 28 & $17,28 *$ \\
\hline setembro & 9 & 90,00 & 1 & 10,00 & 10 & $6,40 *$ \\
\hline outubro & 8 & 100,00 & 0 & 0,00 & 8 & $8,00 *$ \\
\hline novembro & 13 & 68,42 & 6 & 31,58 & 19 & 2,58 \\
\hline dezembro & 6 & 50,00 & 6 & 50,00 & 12 & 0,00 \\
\hline fevereiro & 15 & 75,00 & 5 & 25,00 & 20 & $5,00 *$ \\
\hline maio & 9 & 52,94 & 8 & 47,06 & 17 & 0,06 \\
\hline junho & 24 & 82,76 & 5 & 17,24 & 29 & $12,44^{*}$ \\
\hline julho/2002 & 6 & 85,71 & 1 & 14,29 & 7 & 3,58 \\
\hline TOTAL & 115 & 76,67 & 35 & 23,33 & 150 & $42,66^{*}$ \\
\hline
\end{tabular}

período reprodutivo, a manutenção da proporção sexual de 1:1 é provavelmente mais importante para os bagres em que os machos carregam ovos ou embriões na cavidade oral (região hióide). Segundo esses autores, as fêmeas predominam em espécies de bagres marinhos nas quais a participação dos machos na reprodução é limitada à fertilização dos ovos, como parece ser o caso de $H$. proops. Assim, se a estrutura em sexo varia também ao longo do ciclo de vida dos peixes, análises desse tipo podem subsidiar estudos de avaliação do potencial reprodutivo e de estimativas de tamanho de estoque (VAZZOLER, 1996).

A análise da relação peso/comprimento tem sido utilizada para estimativas de biomassa a partir de dados de freqüência de comprimento e como medida da variação do peso esperado para o comprimento de um indivíduo, indicando sua condição (o acúmulo de gordura e desenvolvimento gonadal) (Rossi-WongTSCHOWSKI, 1977).

Os dados de peso e comprimento de cada indivíduo foram plotados em gráficos de dispersão (Fig. 2), ajustando-se os parâmetros por regressão não-linear pelo método dos mínimos quadrados. A relação encontrada foi $\mathrm{Wt}=2,44 \times 10^{-6} \cdot \mathrm{Lt}^{3,544}$ para fêmeas (Fig. $2 \mathrm{~A}$ ), e $\mathrm{Wt}=$ $2 \times 10^{-6} \cdot \mathrm{Lt}^{3,252}$ para machos (Fig. 2B). O coeficiente de alometria, relacionado à forma de crescimento dos indivíduos, apresentou diferenças significativas entre os sexos (ANCOVA; $p<0,05$ ), assim como o parâmetro "a", relacionado ao grau de engorda. A população de $H$. proops estudada apresentou crescimento alométrico positivo, o que coincide com o que foi encontrado para outras espécies de bagres marinhos, como Netuma barba (=Genidens barbus (Lacepède, 1803)) em ReIs (1986) e Genidens genidens (Valenciennes, 1839) em OliveIRA \& Novelli (2005) e PEDRA et al. (2006).

Segundo Araújo \& Vicentini (2001), valores inferiores ou superiores a 3 indicam indivíduos que, ao longo do crescimento, se tornam mais "longilíneos" ou "redondos", respectivamente. De acordo com essa escala, $H$. proops (principalmente as fêmeas - coeficiente de alometria maior) apresentaria formato do corpo mais arredondado, o que é comum entre os Siluriformes (Figueiredo \& Menezes, 1978). O desenvolvimento das gônadas femininas ocorre em ritmo mais intenso que o incremento em comprimento ou em peso do peixe (daí a mudança mais acentuada da forma do corpo das fêmeas ao longo do ciclo de vida) (IsAAC-NAHUM \& VAZZOLER, 1983). LeCOMte et al. (1989) demonstraram que, em estuários da Guiana Francesa, H. proops cresce rapidamente durante os dezoito primeiros meses e apresenta uma pequena longevidade, sendo que as fêmeas são maiores e têm maior expectativa de vida do que os machos.

A análise da variação mensal dos valores médios do índice gonadossomático revelou que $H$. proops apresenta período reprodutivo entre os meses de outubro e maio, com pronunciado pico da atividade em fevereiro (Fig. 3). Embora as tendências na variação desse índice sejam semelhantes entre os sexos, nos machos os valores médios mensais são menores (Fig. 3) (desenvolvimento gonadal mais marcante nas fêmeas). De acordo com Chaves (1991), as fêmeas realmente são melhores indicadoras do período de reprodução. Um único e amplo 
período reprodutivo parece comum entre os Ariidae, em geral com início na primavera e estendendo-se até o fim do verão (RIMMER \& MERRICK, 1983; REIS, 1986; BARBIERI et al., 1992; Melo \& TeIXeIra, 1992; Gomes \& Araújo, 2004; FÁvAro et al., 2005).

Fatores como o peso das gônadas e do estômago podem afetar as variações das condições gerais do "bemestar" do peixe, visto que dependem do grau de desenvolvimento gonadal e da atividade alimentar por ocasião da sua captura (Agostinho et al., 1990). Por essa razão o fator de condição é um índice muito utilizado para revelar variações sazonais em parâmetros biológicos, já que está relacionado com ciclos gonadais e alimentares, dentre outros (Goulart \& Verani, 1992). Em nosso estudo, o fator de condição das fêmeas revelou decréscimo em outubro, início do período reprodutivo (esse resultado não pôde ser confirmado para os machos, já que não foram capturados indivíduos desse sexo em outubro). Para os machos, observa-se decréscimo na condição geral dos indivíduos no final do período reprodutivo. É importante observar também que os valores médios mensais do fator de condição para as fêmeas foram evidentemente mais baixos que os dos machos (Fig. 3). Essa diferença pode ser justificada pelo alto investimento das fêmeas na produção de gametas. De acordo com ReIs (1986), os Ariidae possuem os maiores ovócitos dentre os Osteichthyes. Além disso, as gônadas maduras de muitos Ariidae ocupam a maior parte da cavidade do corpo, comprimindo o estômago e o intestino e dificultando a alimentação durante o período que antecede a reprodução (RIMMER \& MERRICK, 1983). Esses dois fatores, aliados ao fato de $H$. proops se reproduzir em um período muito longo, podem ser responsáveis pelas baixas condições gerais das fêmeas em relação aos machos.

Como mencionado anteriormente, os machos de algumas espécies de Ariidae coletam os ovos e os carregam em sua cavidade bucal logo após a fertilização com a finalidade de protegê-los até que eclodam (REIs, 1986). Para aumentar o espaço disponível para carregar ovos, a região hióide desses machos se expande quando

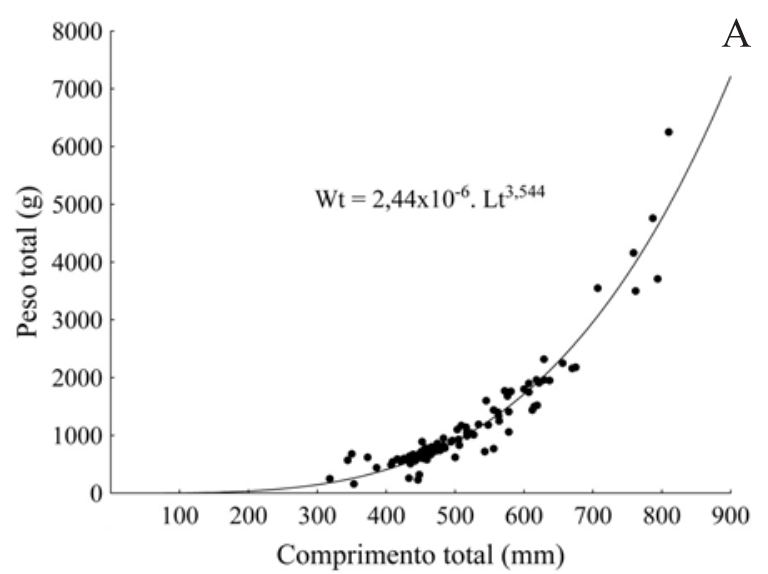

se aproxima o período reprodutivo. Genidens genidens é um exemplo de bagre marinho em que os machos transportam os ovos dentro da cavidade bucal. Estudando essa espécie, BARBIERI et al. (1992) verificaram que ambos os sexos mostraram baixa condição do corpo durante o período reprodutivo, sendo que na maioria das vezes a condição dos machos foi notavelmente menor. Como os ovos incubados ocupam completamente a cavidade orofaringeal, os machos provavelmente cessam a alimentação por completo durante o período de incubação.

Para $H$. proops, não foi encontrado registro de qualquer tipo de cuidado parental realizado pelos machos. Além disso, não foi observada a referida expansão da região hióide. Se de fato esse cuidado parental não ocorre em $H$. proops, há mais uma razão para que os valores de condição geral dos machos sejam superiores àqueles das fêmeas (Fig. 3).

A maturação das gônadas e/ou atividade reprodutiva implicam na utilização de materiais obtidos a partir do alimento ingerido e, principalmente, de reservas energéticas depositadas em diferentes partes do organismo, sendo legítimo esperar que o peso do fígado e outros órgãos de reserva reflitam esse fato (AgostinHo et al., 1990). Os resultados do índice hepatossomático de fêmeas mostram um aumento no início do período reprodutivo e, logo em seguida, uma nítida depleção no peso do fígado, evidenciando que a gordura acumulada no referido órgão foi gasta nas atividades reprodutivas (Fig. 3). Segundo LARSON (1974), as modificações no fígado durante o desenvolvimento gonadal têm sido associadas mais ao fornecimento de precursores vitelínicos do que à mobilização de reservas energéticas. Dessa maneira, os machos não apresentariam acúmulo de gordura no fígado tanto quanto às fêmeas. Isso não pôde ser confirmado para $H$. proops porque o baixo número de machos na amostra impossibilitou a visualização de um padrão claro na variação do índice hepatossomático (Fig. 3B).

$\mathrm{O}$ índice de repleção, por apresentar um caráter quantitativo, pode fornecer informações mais seguras para indicar o período em que os peixes apresentam maior atividade alimentar (BARBIERI, 1992). A análise desse parâmetro sugere que tanto fêmeas como machos

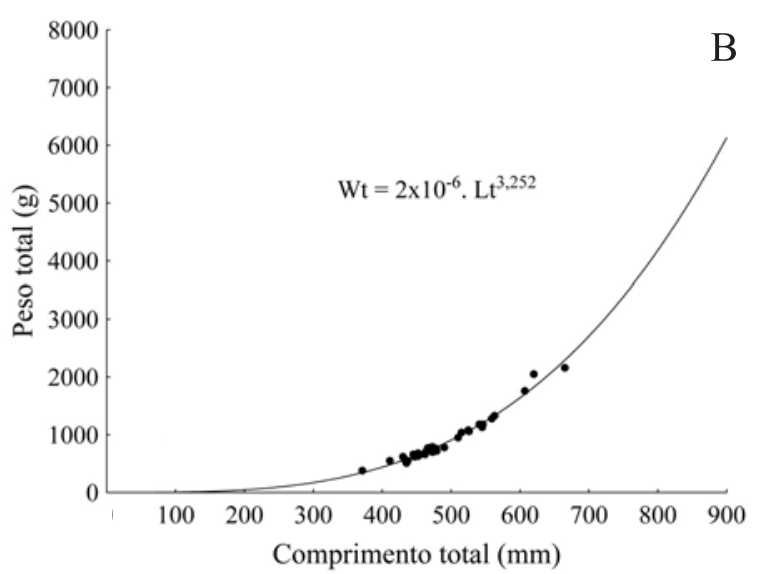

Fig. 2. Relação peso total/comprimento total para fêmeas (A) e para machos (B) de Hexanematichithys proops (Valenciennes, 1840) capturados no litoral ocidental maranhense de julho de 2001 a julho de 2002 . 
apresentam melhores condições alimentares nos meses que antecedem e sucedem o período reprodutivo (outubro a maio) (Fig. 4). Antes da reprodução, o aumento na tomada de alimento ocasiona acentuado acúmulo de gordura, conforme indicado nas análises do índice hepatossomático (Fig. 3A). Esses resultados confirmam uma nítida variação cíclica envolvendo a reprodução e alimentação (BRAGA, 1990).

O volume das gônadas prontas para desovar é uma das principais causas físicas que impedem ou limitam a ingestão de alimento (Zavala-CAmin, 1996). Assim, muitas espécies se alimentam menos intensamente durante a reprodução, o que seria a causa da queda no índice de repleção para fêmeas nesse período (BARBIERI \& BARBIERI, 1984).

FONTELES-FILHO (1989) considera que a estratégia reprodutiva deve estar em total consonância com a estratégia alimentar pois uma das condições essenciais para o equilíbrio populacional é a manutenção do suprimento alimentar. Através deste, as funções de predação e competição podem ser controladas e os indivíduos podem ter o desenvolvimento adequado para manter o equilíbrio estrutural da população.
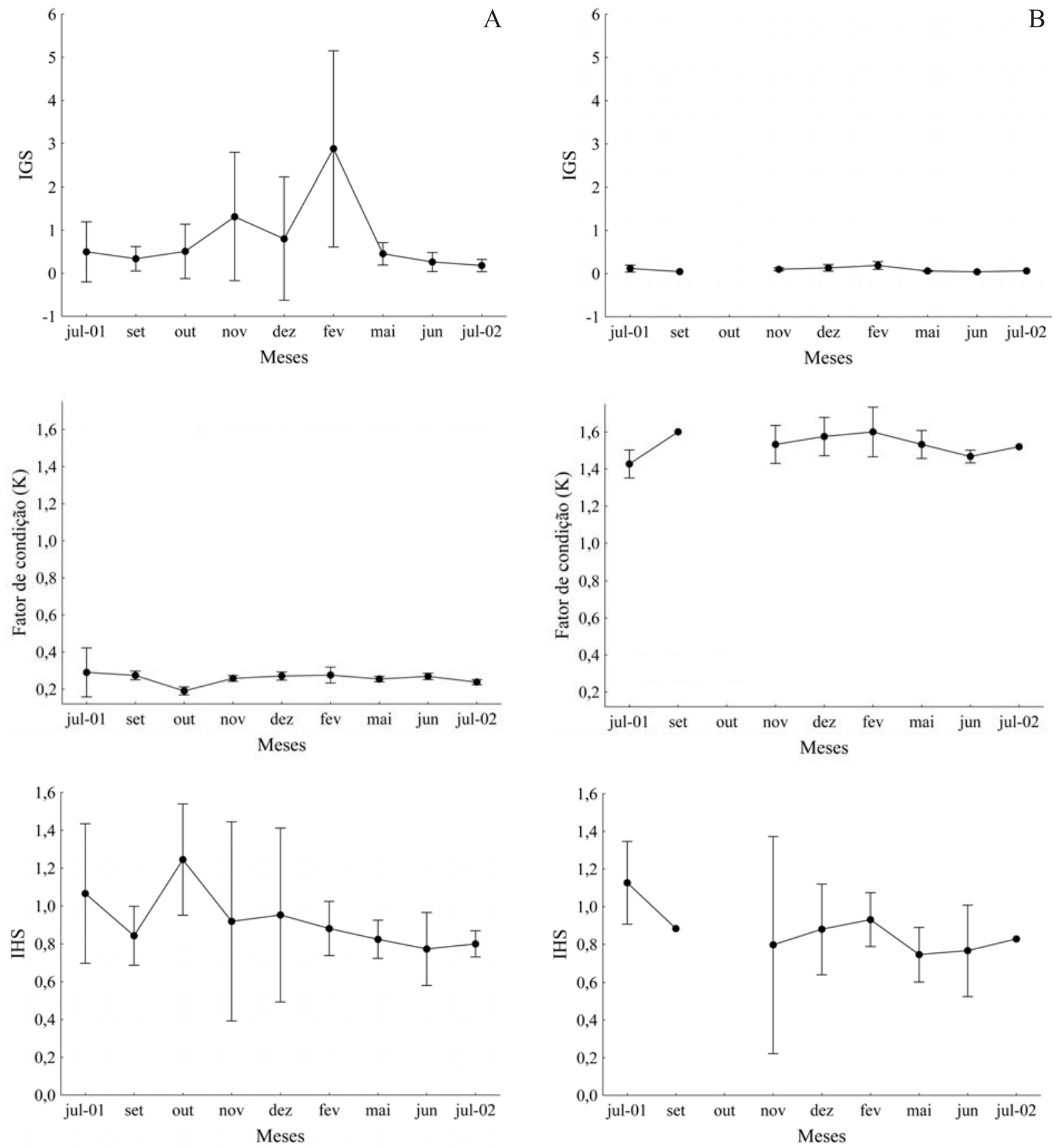

Fig. 3. Variação mensal das médias do índice gonadossomático (IGS), fator de condição e índice hepatossomático (IHS) de fêmeas (A) e machos (B) de Hexanematichithys proops (Valenciennes, 1840) capturados no litoral ocidental maranhense de julho de 2001 a julho de 2002. As barras verticais representam o desvio padrão sobre as médias. 

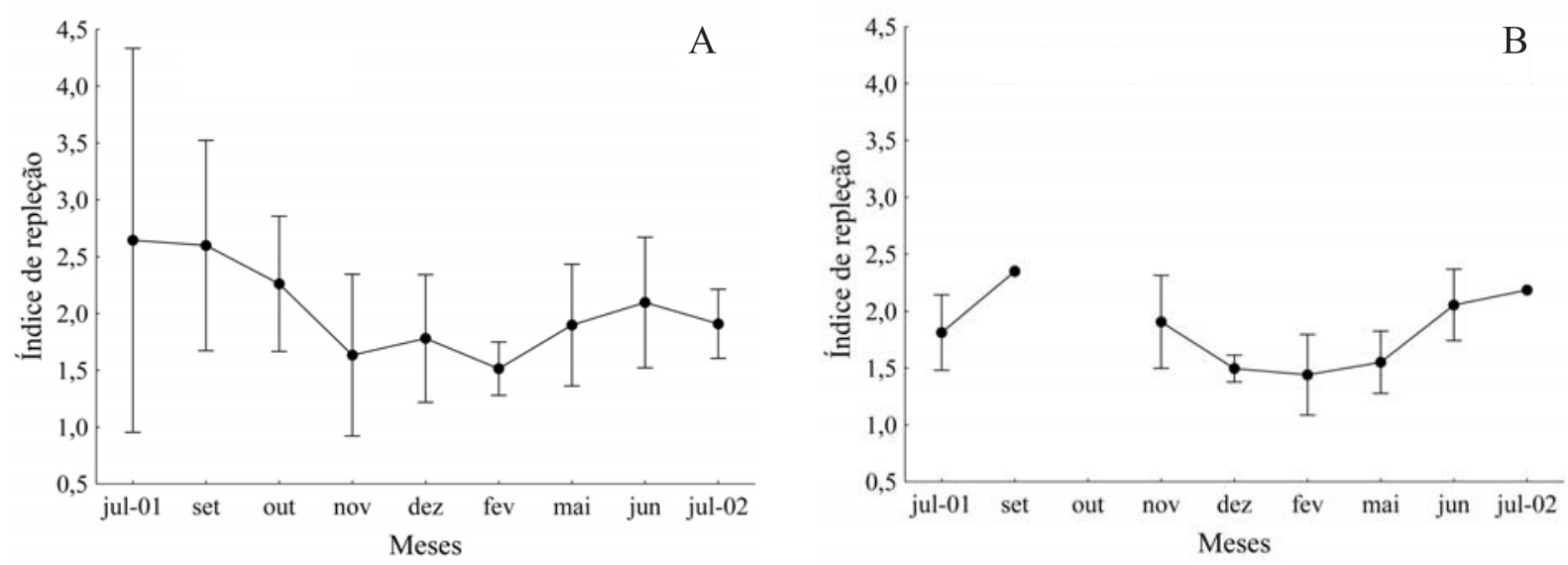

Fig. 4. Variação mensal das médias do índice de repleção para fêmeas (A) e machos (B), de Hexanematichithys proops (Valenciennes, 1840) capturados no litoral ocidental maranhense de julho de 2001 a julho de 2002. As barras verticais representam o desvio padrão sobre as médias.

Agradecimentos. Agradecemos ao Conselho de Aperfeiçoamento de Pessoal de Nível Superior (CAPES) pela concessão de bolsa, ao Programa REVIZEE - SCOR/NORTE pela coleta de material biológico e aos revisores deste artigo pelas valiosas sugestões.

\section{REFERÊNCIAS BIBLIOGRÁFICAS}

Agostinho, A. A.; Barbieri, G. \& Verani, J. R. V. 1990. Variação do fator de condição e do índice hepatossomático e suas relações com o ciclo reprodutivo em Rhinelepis aspera (Agassiz, 1829) (Osteichthyes, Loricariidae) no Rio Paranapanema, Porecatu, PR. Ciência e Cultura 42(9):711-714.

Andreata, J. V.; Barbieri, L. R. R.; Sebilia, A. S. B.; Silva, M. H. C. \& Santos, R. P. 1989. A list of Marapendi Lagoon fishes, Rio de Janeiro, Brazil. Atlântica 11:5-17.

AraúJo, F. G. 1984. Hábitos alimentares de três bagres marinhos (Ariidae) no estuário da Lagoa dos Patos (RS), Brasil. Atlântica 7:47-63

ARAúJo, F. G. 1988. Abundância relativa, distribuição e movimentos sazonais de bagres marinhos (Siluriformes, Ariidae) no estuário da Lagoa dos Patos, RS. Revista Brasileira de Zoologia 5(4):509-543.

AraúJo, F. G. \& Vicentini, R. N. 2001. Relação peso-comprimento da corvina Micropogonias furnieri (Desmarest) (Pisces, Sciaenidae) na Baía de Sepetiba, Rio de Janeiro. Revista Brasileira de Zoologia 18(1):133-138.

Azevedo, M. C. C.; Araújo, F. G.; Cruz-Filho, A. G.; Gomes, I. D. \& Pessanha, A. L. G. 1999. Variação espacial e temporal de bagres marinhos (Siluriformes, Ariidae) na Baía de Sepetiba, Rio de Janeiro. Revista Brasileira de Biologia 59(3):443-454.

BARBIERI, G. 1992. Dinâmica da nutrição de Astyanax scabripinnis paranae (Characiformes, Characidae) do Ribeirão do Fazzari: São Carlos, SP. Revista da Sociedade Brasileira de Zoologia 21(1):68-72.

BARBIERI, G. \& BARBIERI, M. C. 1984. Note on nutritional dynamics of Gymnotus carapo (L.) from the Lobo Reservoir, São Paulo State, Brazil. Journal of Fish Biology 24:351-355.

Barbieri, G.; Santos, R. P. \& Andreata, J. V. 1992. Reproductive biology of the marine catfish, Genidens genidens (Siluriformes, Ariidae), in the Jacarepaguá Lagoon system, Rio de Janeiro, Brazil. Environmental Biology of Fishes 35:23-35.

Braga, F. M. S. 1990. Aspectos da reprodução e alimentação de peixes comuns em um trecho do rio Tocantins entre Imperatriz e Estreito, Estado do Maranhão e Tocantins, Brasil. Revista Brasileira de Biologia 50(3):547-558.

Chaves, P. DE T. DA C. 1991. Testículos: estrutura e dinâmica de maturação. In: Chaves, P. DE T. DA C. ed. Histologia de peixes - I Semana sobre histologia de peixes da FCAVJUNESP. Jaboticabal, FUNEP. p.47-54.

Chaves, P. DE T. DA C. 1994. A incubação de ovos e larvas em Genidens genidens (Valenciennes) (Siluriformes, Ariidae) da
Baía de Guaratuba, Paraná, Brasil. Revista Brasileira de Zoologia 11(4):641-648.

Craig, I. D. H. 1980. Contribuição ao conhecimento da fauna íctica costeira da região de Peruíbe, SP. I: Família Ariidae. Revista Brasileira de Biologia 40(4):755-758.

Dos SAntos, E. P. Dos. 1978. Dinâmica de populações aplicada à pesca e piscicultura. São Paulo, HUCITEC, EDUSP. 130p.

Etchevers, S. L. 1978. Contribution to the biology of the sea catfish, Arius spixii (Agassiz) (Pisces-Ariidae), South of Margarita Island, Venezuela. Bulletin Marine of Science 28:381-385.

Fávaro, L. F.; Frehse, F de A.; Oliveira, R. N. DE \& Júnior, R. S. 2005. Reprodução do bagre amarelo, Cathorops spixii (Siluriformes, Ariidae), da Baía de Pinheiros, região estuarina do litoral do Paraná, Brasil. Revista Brasileira de Zoologia 22(4): 1022-1029.

Figueiredo, J. L. \& Menezes, N. A. 1978. Manual de Peixes Marinhos do Sudeste do Brasil. II Teleostei (1). São Paulo, Museu de Zoologia, Universidade de São Paulo. 110p. Fonteles-Filho, A. A. 1989. Recursos pesqueiros: biologia e dinâmica populacional. Fortaleza, Imprensa Oficial do Ceará. 296p.

Goldberg, D. E. \& Scheiner, S. M. 1993. ANOVA and ANCOVA: Field Competition Experiments. In: Scheiner, S. M. \& Gurevitch, J. eds. Design and Analysis of Ecological Experiments. New York, Chapman \& Hall. p.69-93.

Gomes, I. D. \& Araúso, F. G. 2004. Reproductive biology of two marine catfishes (Siluriformes, Ariidae) in the Sepetiba Bay, Brazil. Revista de Biologia Tropical 52(1):143-156.

Goulart, E. \& Verani, J. R. 1992. Proporção sexual, relação peso/ comprimento e fator de condição de Hypostomus commersonii Valenciennes, 1840 (Osteichthyes, Loricariidae) da represa Capivari-Cachoeira, Paraná, Brasil. Revista Unimar 14 (Suplemento):19-33.

Guedes, D. de S. \& Vasconcelos Filho, A. De L. 1980. Estudo ecológico da região de Itamaracá, Pernambuco, Brasil. IX. Informações sobre alimentação dos bagres branco e amarelo (Pisces, Ariidae). Trabalhos Oceanográficos da Universidade Federal de Pernambuco 15:323-330.

IsaAC-Nahum, V. J. \& VAzzoler, A. E. A. DE M. 1983. Biologia reprodutiva de Micropogonias furnieri (Desmarest, 1823) (Teleostei, Scianidae), 1. Fator de condição como indicador do período de desova. Boletim do Instituto Oceanográfico 32(1):63-69.

LARSON, G. L. 1974. Liver weight of brook trout in a highmontain lake in Washington State. Progressive FishCulturist 35:234-236.

Lecomte, F.; Meunier, F. J. \& Rojas-Beltran, R. 1986. Donnes preliminiares sur la croissance de deux téléostéens de Guyane, Arius proops (Ariidae, Siluriforme) et Leporinus friderici (Anostomidae, Characoidei). Cybium 10(2):121-134. 
Lecomte, F.; Meunier, F. J. \& Rojas-Beltran, R. 1989. Some data on the growth of Arius proops (Ariidae, Siluriforme) in the estuaries of French Guyana. Aquatic Living Resource 2:63-68.

LE-Cren, E. D. 1951. The lenght-weight relationship and seasonal cycle in gonad weight and conditions in the perch Perca fluviatilis. Journal Animal Ecology 20(2):201-219.

Melo, S. C. \& TeiXeiRA, R. L. 1992. Distribuição, reprodução e alimentação de Cathorops spixii e Arius rugispinis (Pisces: Ariidae) do Complexo Mundaú/Manguaba, Maceió-AL. Revista Brasileira de Biologia 52(1):169-180.

Meunier, F. J.; Rojas-Beltran, R.; Boujard, T. \& Lecomte, F. 1994. Rythmes saisonniers de la croissance chez quelques Téléostéens de Guyane française. Revue D'Hydrobiologie Tropicale 27(4):423-440.

Mishima, M. \& TAnj, S. 1981. Distribuição geográfica dos bagres marinhos (Osteichthyes, Ariidae) no complexo estuarino lagunar de Cananéia $\left(25^{\circ} \mathrm{S}, 48^{\circ} \mathrm{W}\right)$. Boletim do Instituto de Pesca 8:157-172.

1982. Nicho alimentar de bagres marinhos (Teleostei, Ariidae) no complexo estuarino lagunar de Cananéia $\left(25^{\circ} \mathrm{S}\right.$, $\left.48^{\circ} \mathrm{W}\right)$. Boletim do Instituto de Pesca 9:131-140.

Moyle, P. B. \& CeCH-JR, J. J. 1988. Fishes - An Introduction to Ichthyology. New Jersey, Englewood Clifs. 559p.

Myers, R. H. 1990. Classical and modern regression with applications. Belmont, Duxbury. 488p.

Nikolsky, G. V. 1963. The Ecology of Fishes. London, Academic. $352 \mathrm{p}$

1969. Theory of fish population dynamics. Edinburgh, Oliver \& Boyd. 323p.

Oliveira, M de A. \& Novelli, R. 2005. Idade e crescimento do bagre Genidens genidens na Baía da Lagoa do Açu, norte do Estado do Rio de Janeiro. Tropical Oceanography 33(1):57-66.

Pedra, M. L. R.; Oliveira, M. De A. \& Novelli, R. 2006. Biologia alimentar do bagre Genidens genidens (Valenciennes, 1839) na Barra da Lagoa do Açu, norte do Estado do Rio de Janeiro.
Acta Biologica Leopondensia 28(1):38-41.

Planquette P. \& Rojas-Beltran, R. 1984. Étude de l'impact du projet d'aménagement de Petit-Saut (Guyane) sur de peuplement ichthyologique. État d'avancement dês travaux. Cayenne, Laboratoire d'Hydrobiologie, INRA Guyane, rapport pour l'EDF. 68p

REIS, E. G. 1986. Reproduction and feeding habits of the marine catfish Netuma barba (Siluriformes, Ariidae) in the estuary of Lagoa dos Patos, Brazil. Atlântica 8:35-55.

Reis, R. E; Kullander, S. O. \& Ferraris, Jr. C. J. 2003. Check list of the freshwater fishes of South and Central America. Porto Alegre, EDIPUCRS. 729p.

Rimmer, M. A. \& Merrick, J. R. 1983. A review of reproduction and development in the fork-tailed catfishes (Ariidae). Proceedings of the Limnology Society 107:41-50.

Rojas-Beltran, R. 1989. Quelques aspects de l'écologie alimentaire de trois mâchoirans (Teleostei, Siluriformes, Ariidae) de la Guyane. Cybium 13(2):181-187.

Rossi-Wongtschowski, C. L. B. 1977. Estudo das variações da relação peso total/comprimento total em função do ciclo reprodutivo e comportamento de Sardinella brasiliensis (Steindachner, 1879) da costa do Brasil entre $23^{\circ} \mathrm{S}$ e $28^{\circ} \mathrm{S}$. Boletim do Instituto Oceanográfico 26:131-180.

Statsoft, Inc. 2005. Statistica (data analysis software system), version 7.1.

VAZzoler, A. E. A. DE M. 1996. Biologia da reprodução de peixes teleósteos: teoria e prática. Maringá, EDUEM. 169p.

Vazzoler, A. E. A. de M. Caraciolo-Malta, M. C. \& Amadio, S. A. 1989. Aspectos biológicos de peixes amazônicos. XII. Indicadores quantitativos do período de desova das espécies do gênero Semaprochilodus (Characiformes, Prochilodontidae) do baixo rio Negro, Amazonas, Brasil. Revista Brasileira de Biologia 49(1): 175-181.

Zavala-CAmin, L. A. 1996. Introdução aos estudos sobre alimentação natural em peixes. Maringá, EDUEM. 129p.

Recebido em novembro de 2005. Aceito em julho de 2007. ISSN 0073-4721

Artigo disponível em: www.scielo.br/isz 